\title{
Study of angular momentum hindrance in heavy ion fusion reactions
}

\author{
Ajay Kumar ${ }^{1, a}$, A. Kumar ${ }^{2}$, B.R. Behra ${ }^{2}$, Hardev Singh $^{3}$, R.P. Singh ${ }^{4}$, R. Kumar ${ }^{4}$ and K.S. Golda ${ }^{4}$ \\ ${ }^{1}$ Department of Physics, Banaras Hindu University, Varanasi 221005, India \\ ${ }^{2}$ Department of Physics, Panjab University, Chandigarh 160014, India \\ ${ }^{3}$ Department of Physics, Kurukshetra University, Kurukshetra 136118, India \\ ${ }^{4}$ IUAC, New Delhi 110067, India
}

\begin{abstract}
The systematic study of the properties of hot nuclei by detecting the emitted charged particles and neutrons in coincidence with residual nuclei provides very critical information about its nuclear level density. These emitted particles capable to explain the behavior of the nucleus at various stages of the de-excitation cascade process. So, we have studied, a set of four compound nuclei, which were populated by mass-symmetric and mass-asymmetric channels, leading to the same compound nuclei, namely ${ }^{80} \mathrm{Sr}^{*},{ }^{79} \mathrm{Se}^{*},{ }^{76} \mathrm{Kr}^{*}$ and ${ }^{58} \mathrm{Ni}^{*}$ at same excitation energies, respectively and found that the experimental neutron and charged particle spectra for symmetric channel show deviations at higher energies in comparison to the statistical model calculations.
\end{abstract}

\section{Introduction}

The statistical model has been used for many decades to analyze a variety of observables related to the compound nucleus decay. So the successful description of the particle emission remains essential for evaluating the validity of the model and the choice of the parameters within it. In the case of the compound nuclei at moderate energies and angular momenta, such as those produced with light ion projectiles, the experimental charged particle spectra are well explained in terms of the statistical model, employing the optical model transmission coefficients. However, in the case of heavy ion induced fusion reactions, there have been several claims of serious discrepancies between the predictions of the standard statistical model and the measured light charged particle energy spectra [1-4]. Measured light charged particle spectra have been characterized as having lower energy than predicted. It is also observed that these spectra may be well explained in terms of the statistical model incorporating only a spin dependent level density and without lowering the emission barriers [5].

In order to confirm these observations, we have studied four compound systems $\left({ }^{80} \mathrm{Sr}^{*},{ }^{79} \mathrm{Se}^{*},{ }^{76} \mathrm{Kr}^{*}\right.$ and ${ }^{58} \mathrm{Ni}^{*}$ ), through mass-symmetric channel and massasymmetric channels at same excitation energies, respectively.

\section{Experiment}

The data were obtained using 15 UD Pelletron at Inter University Accelerator Centre (IUAC), New Delhi, India. Pulsed beam of different energies were used to form four compound nuclei using different targets. The experiment was done using the $1.5 \mathrm{~m}$ diameter stainless steel general purpose scattering chamber available at IUAC. Neutron detectors having liquid scintillator cells of BC501 of 12.5 $\mathrm{cm}$ diameter and thicknesses of $12.5 \mathrm{~cm}$ were used at different angles with respect to the beam direction. The neutron energy was determined by the time-of-flight technique. The time-of-flight spectra thus obtained were converted into neutron energy and the intensity was normalized using the neutron detection efficiency code MODEFF [6]. We have also measured the chargedparticle spectra during this experiment using $\Delta E-E$ (40 $\mu \mathrm{m}-5 \mathrm{~mm}$ ) detector telescopes.

\section{Analysis}

The statistical model CASCADE [7] and Dynamical trajectory model calculations (HICOL) [8] were used to perform theoretical calculations.

\section{Results and Discussion}

The neutron spectra of the composite system ${ }^{76} \mathrm{Kr}^{*}$ and ${ }^{58} \mathrm{Ni}$ formed through the asymmetric reaction $\left({ }^{12} \mathrm{C}+{ }^{64} \mathrm{Zn}\right)$ and $\left({ }^{12} \mathrm{C}+{ }^{46} \mathrm{Ti}\right)$ are shown in Fig. 1(a) and 2(a) respectively for different angles with respect to the beam direction. The neutron emission spectra for the comparatively mass-symmetric $\left({ }^{31} \mathrm{P}+{ }^{45} \mathrm{Sc}\right)$ and $\left({ }^{31} \mathrm{P}+{ }^{27} \mathrm{Al}\right)$ systems at different angles for the excitation energies 75.0 and $79.5 \mathrm{MeV}$ respectively are shown in Figs. 1(b) and 2(b). As is clear from the figures, these neutron spectra are not in agreement with the statistical model predictions using normal parameters as used for the asymmetric system. It is also clear that the high energy part of the neutron spectra in the case of a symmetric

\footnotetext{
${ }^{a}$ Corresponding author: atyagi44@yahoo.co.in
} 
system are harder than the statistical model predictions, indicating neutron evaporation at a higher temperature. The slope of the high-energy part of the neutron spectra is very sensitive to the level density $\left(\rho \approx \exp \left[2(\mathrm{aE})^{1 / 2}\right]\right)$ and thus on the level density parameter $a$.

In order to verify quantitatively the experimental trends, the statistical model calculation was performed by changing the value of the level density parameter $a=\mathrm{A} / 10 \mathrm{MeV}^{-1}$. Results of these calculations are shown in Fig. 3(a) and 3(b) for the comparatively mass-symmetric $\left({ }^{31} \mathrm{P}+{ }^{45} \mathrm{Sc}\right)$ and $\left({ }^{31} \mathrm{P}+{ }^{27} \mathrm{Al}\right)$ systems for the excitation energies 75.0 and $79.5 \mathrm{MeV}$ respectively.
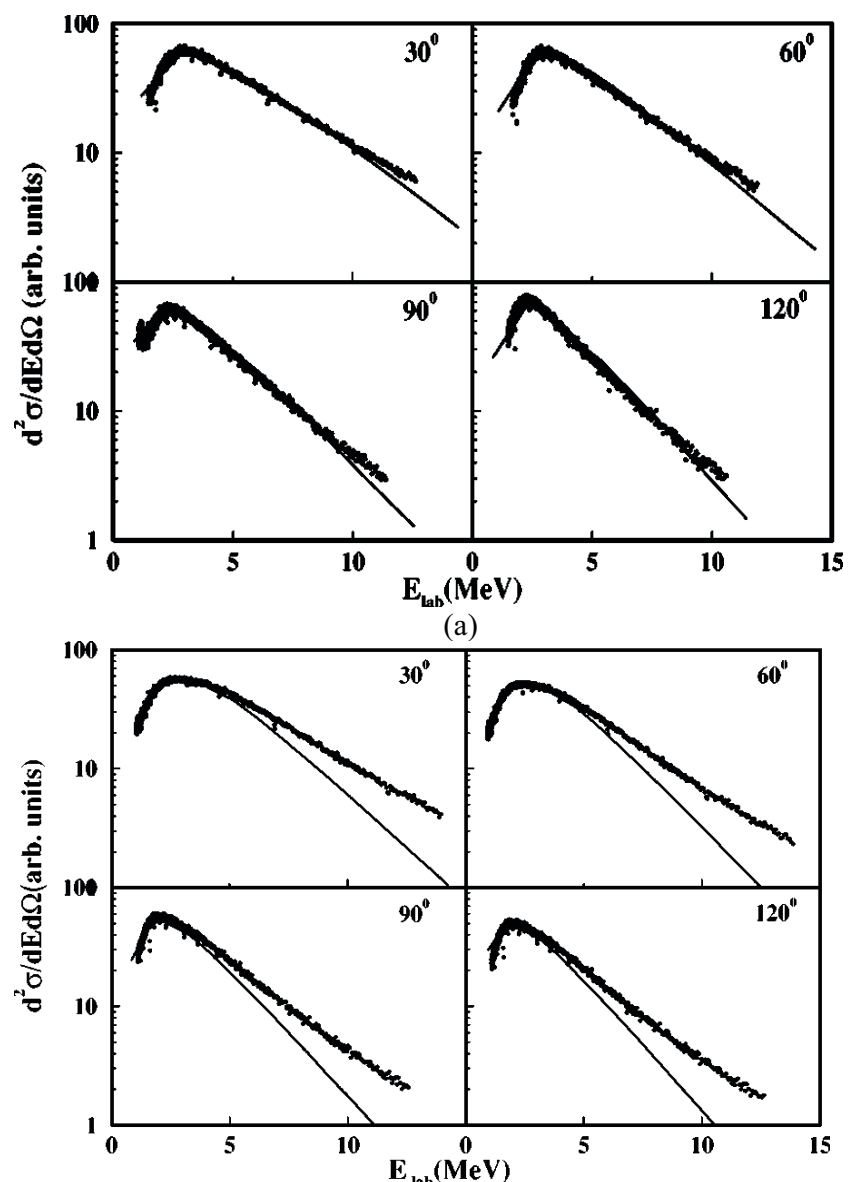

(b)

Figure 1. Comparison of the experimental neutron spectra (dots) with the statistical model (solid line) (a) using $r_{0}=1.25$ and $a=\mathrm{A} / 8$ for the asymmetric reaction ${ }^{12} \mathrm{C}+{ }^{64} \mathrm{Zn}$ with $\ell_{\max }=$ $41 \hbar$ and $\mathrm{E}^{*}=75 \mathrm{MeV}$ at $\mathrm{E}_{\mathrm{lab}}=85 \mathrm{MeV}$ (b) $r_{0}=1.25$ and $a=\mathrm{A} / 8$ for the symmetric reaction ${ }^{31} \mathrm{P}+{ }^{45} \mathrm{Sc}$ with $\ell_{\max }=43 \mathrm{~h}$ and $\mathrm{E}^{*}=75 \mathrm{MeV}$ at $\mathrm{E}_{\mathrm{lab}}=120 \mathrm{MeV}$.

This provides a reasonable description of the data and reproduces the shape of the spectra very well. The lower value of the level density parameter $a=A / 10 \mathrm{MeV}^{-1}$ manifests an effective higher nuclear temperature $[T=\sqrt{ }(E / a)]$ for the neutron evaporation in the case of the mass-symmetric system. We have also compared the experimental particle spectra for two other compound nuclei $\left({ }^{80} \mathrm{Sr}^{*}\right.$ and $\left.^{79} \mathrm{Se}^{*}\right)$ and found same kind of observations.
In order to understand the behavior of nuclear reaction dynamics more clearly, we have also compared the experimental charged-particle spectra for ${ }^{76} \mathrm{Kr}^{*}$ with statistical model calculations using the normal-level density parameter $a=\mathrm{A} / 8$ for alpha and protons in Figs. 4(a) and 4(b) for asymmetric and symmetric systems.

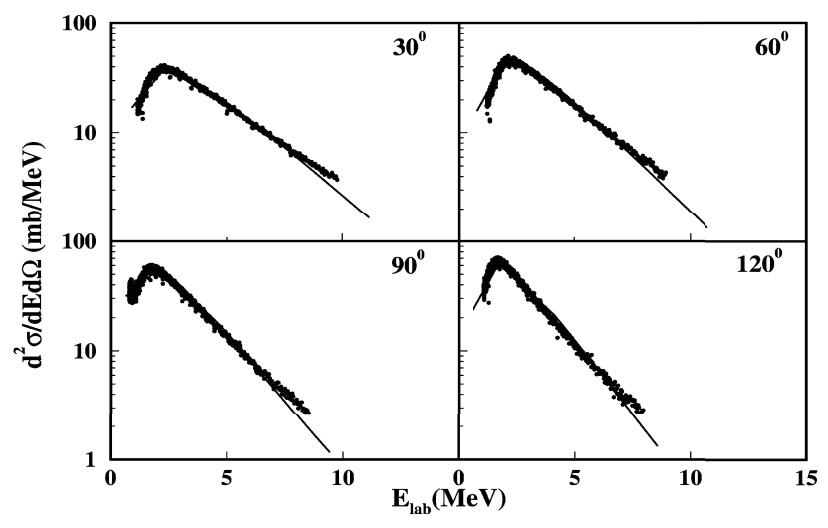

(a)

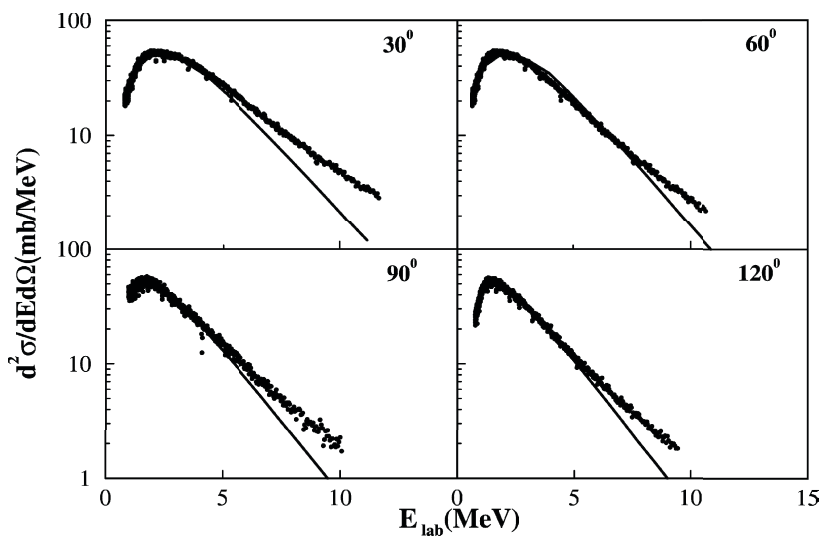

(b)

Figure 2. Comparison of the experimental neutron spectra (dots) with the statistical model (solid line) (a) using $r_{0}=1.25$ and $a=\mathrm{A} / 8$ for the asymmetric reaction ${ }^{12} \mathrm{C}+{ }^{46} \mathrm{Ti}$ with $\ell_{\max }=42.5 \hbar$ and $\mathrm{E}^{*}=79.5 \mathrm{MeV}$ at $\mathrm{E}_{\mathrm{lab}}=80 \mathrm{MeV}$ (b) $r_{0}=1.25$ and $a$ $=\mathrm{A} / 8$ for the symmetric reaction ${ }^{31} \mathrm{P}+{ }^{27} \mathrm{Al}$ with $\ell_{\max }=56.2 \mathrm{~h}$ and $\mathrm{E}^{*}=79.5 \mathrm{MeV}$ at $\mathrm{E}_{\mathrm{lab}}=131 \mathrm{MeV}$.

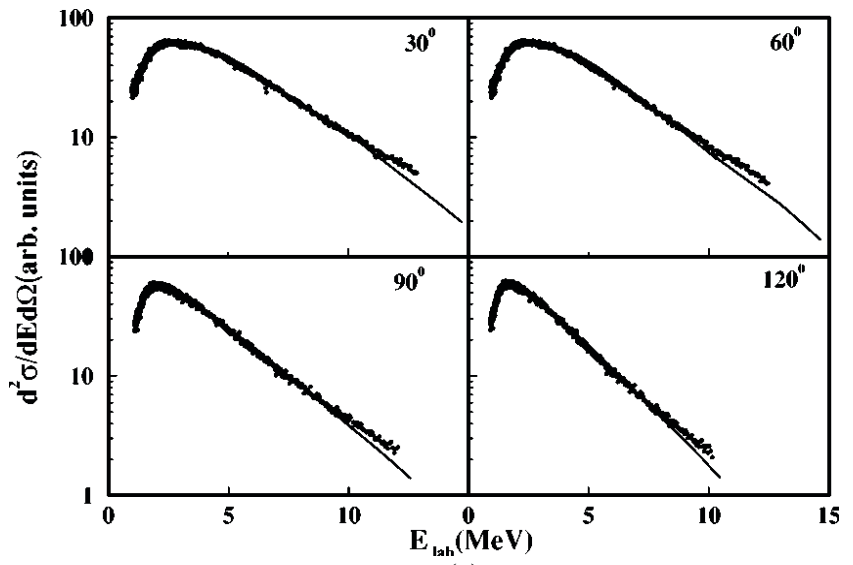

(a) 


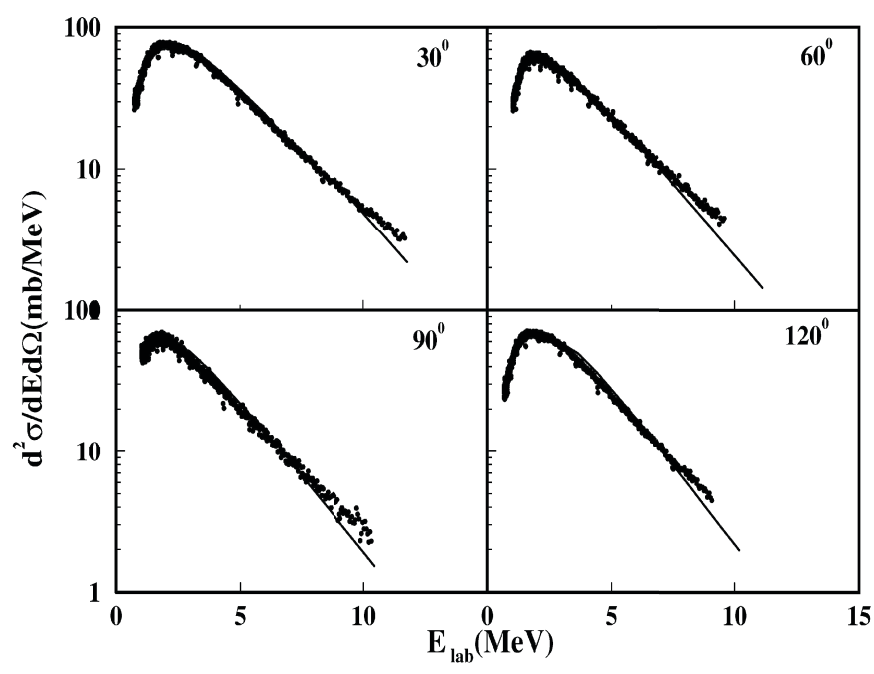

(b)

Figure 3. (a) Comparison of the experimental neutron spectra (dots) with the statistical model (solid line), using $r_{0}=1.25$ and $a$ $=\mathrm{A} / 10$ for ${ }^{31} \mathrm{P}+{ }^{45} \mathrm{Sc}$ with $\ell_{\max }=43 \mathrm{~h}$ and $\mathrm{E}^{*}=75 \mathrm{MeV}$ at $\mathrm{E}_{\mathrm{lab}}=120$ $\mathrm{MeV}$ (b) for ${ }^{31} \mathrm{P}+{ }^{27} \mathrm{Al}$ with $\ell_{\max }=56.2 \mathrm{~h}$ and $\mathrm{E}^{*}=79.5 \mathrm{MeV}$ at $\mathrm{E}_{\mathrm{lab}}=131 \mathrm{MeV}$, using $r_{0}=1.25$ and $a=\mathrm{A} / 10$.

In the case of the charged particle spectra, the experimental data is very consistent with statistical model
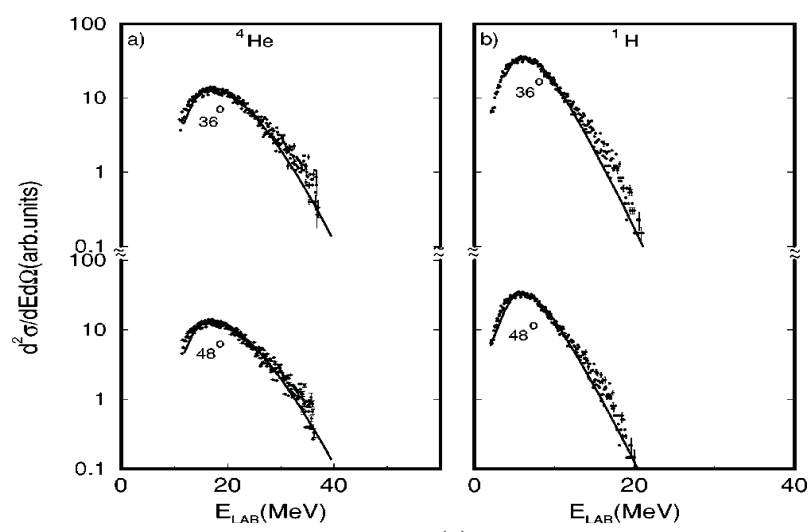

(a)
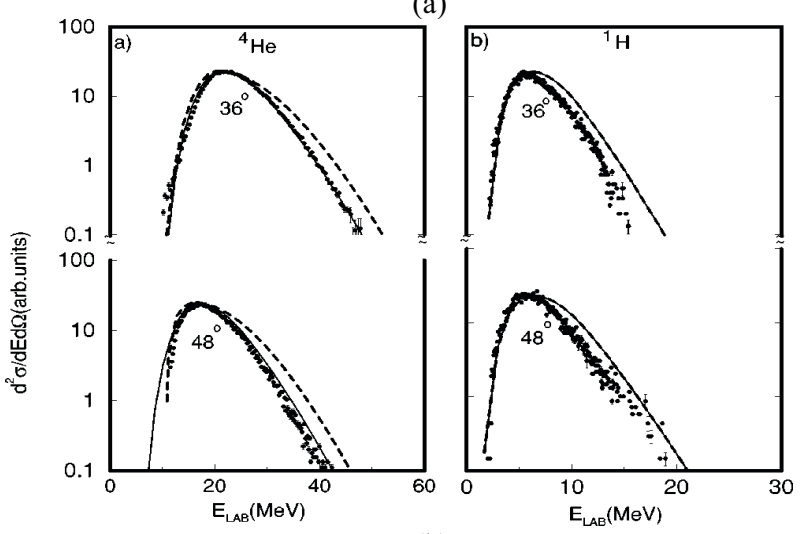

(b)

Figure 4. Comparison of the experimental alpha and proton spectra with the statistical model (solid line) using the transmission coefficients for the spherical nuclei and the RLDM moment of inertia for the (a) asymmetric reaction ${ }^{12} \mathrm{C}+{ }^{64} \mathrm{Zn}$ with $\ell_{\max }=41 \mathrm{~h}$ and $\mathrm{E}^{*}=75 \mathrm{MeV}$ at $\mathrm{E}_{\mathrm{lab}}=85 \mathrm{MeV}$ (b)for ${ }^{31} \mathrm{P}+{ }^{45} \mathrm{Sc}$ with $\ell_{\max }=43 \hbar$ and $\mathrm{E}^{*}=75 \mathrm{MeV}$ at $\mathrm{E}_{\mathrm{lab}}=120 \mathrm{MeV}$. calculations for asymmetric system $\left({ }^{12} \mathrm{C}+{ }^{64} \mathrm{Zn}\right)$, while the high energy part of evaporation spectra found to be softer as compared to the theoretical spectra in case of symmetric system $\left({ }^{31} \mathrm{P}+{ }^{45} \mathrm{Sc}\right)$. This is contrary to the neutron spectra, which are found to be harder as compared to the statistical model calculation.
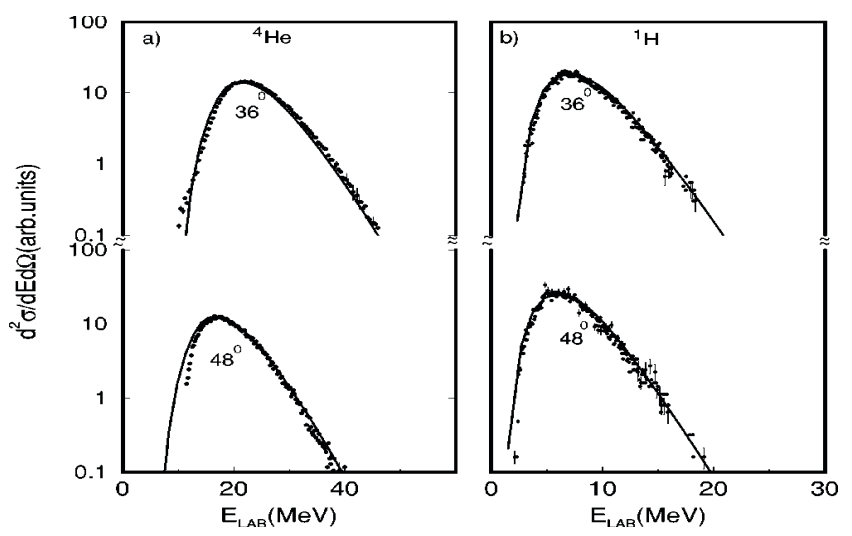

Figure 5. Comparison of experimental spectra with the statistical model for the reaction ${ }^{31} \mathrm{P}+{ }^{45} \mathrm{Sc}$ using the HICOL predicted $\ell_{\max }=30 \hbar$ (a) $\alpha$-spectra (b) for proton spectra.

The statistical model calculations with the HICOL predicted value of $\ell=30 \mathrm{~h}$ for the symmetric system is compared with experimental alpha and proton spectra in Fig.5. The excellent agreement with theory suggests that the deviation from the statistical model results from dynamical effects arising from the mass symmetry of the entrance channel during the formation of the compound nucleus.

\section{Summary}

Four compound nuclei $\left({ }^{80} \mathrm{Sr}^{*},{ }^{79} \mathrm{Se}^{*},{ }^{76} \mathrm{Kr}^{*}\right.$ and $\left.{ }^{58} \mathrm{Ni}^{*}\right)$ were formed through different asymmetric and symmetric entrance channel at same excitation energy for a corresponding compound system. It was observed that neutron energy spectra for asymmetric systems are well described by statistical model predictions using the normal value of the level density parameter $a=\mathrm{A} / 8$ $\mathrm{MeV}^{-1}$. However, in the case of the symmetric systems, the statistical model interpretation of the data requires a change in the value of $a=\mathrm{A} / 10 \mathrm{MeV}^{-1}$. However the charged particle spectra for the symmetric system is not consistent with the statistical model calculation by using the angular momentum obtained by Bass model and the experimental charged particle data for symmetric system is very consistent with statistical model, when the HICOL predicted angular momentum value is used.

But again if the HICOL predicted angular momentum is used for symmetric system particle spectra (charged particle and neutrons), the neutron spectra becomes harder comparative to statistical model calculation. So to get more clear information about the variation of angular momentum, we need to do more experiments for 
determination of the value of fused angular momentum in both type of systems i.e. symmetric as well as asymmetric system. It looks from the experimental observations that in case of symmetric system, whole angular momentum is not going to fuse and delayed evolution of the compound system for symmetric system is responsible for the deviation of experimental spectra with statistical model calculation.

\section{Acknowledgments}

The authors acknowledge with thanks the Accelerator crew of Inter University Accelerator Centre, New Delhi, for providing a high-quality beam. The first author also acknowledges financial support from SERB, DST, New Delhi, India, through project grant SR/FTP/PS-068/2010. The first author also acknowledges financial support from UGC Networking grant received from Physics Department, BHU, Varanasi.

\section{References}

1. B. Fornal, G. Prete, G. Nebbia, F. Trotti, G. Viesti, D. Fabris, K. Hagel, and J. B. Notwitz, Phys. Rev. C 37, 2624 (1988)

2. B. Fornal, Phys. Rev. C 41, 127 (1990)

3. R. K. Choudhury, P. L. Gonthier, K. Hagel, M. N. Namboodiri, J. B. Natowitz, L. Alder, S. Simon, S. Kniffen, and G. Berkowitz, Phys. Lett. B 143, 74 (1984)

4. G. Viesti, B. Fornal, D. Fabris, K. Hagel, J. B. Natowitz, G. Nebbia, G. Prete, and F. Trotti, Phys. Rev. C 38, 2640 (1988)

5. I. M. Govil, J. R. Huizenga, W. U. Schroder, and J. Toke, Phys. Lett. B 197, 51(1987)

6. R.A. Cecil, B.D. Anderson, and R. Madey, Nucl. Instrum. Methods 161, 439 (1979)

7. F. Puhlhofer, Nucl. Phys. A280, 267 (1997)

8. H. Feldmeier, Rep. Prog. Phys. 50, 915 (1987)

9. R. Bass, Phys. Lett. B 47, 139 (1973) 